\title{
Kein Anspruch von Kardiologen auf die Erbringung von kernspintomografischen Leistungen in der vertragsärztlichen Versorgung
}

Urteil des Landessozialgerichts BerlinBrandenburg vom 20.02.2013, Az.: L 7 KA60/11 -

\section{Einführung}

$\nabla$

Mit Urteil vom 20.02.2013 (Az.: L 7 KA60/11) hat das Landessozialgericht Berlin-Brandenburg entschieden, dass Fachärzte für Innere Medizin und Kardiologie keinen Anspruch auf Erteilung einer Genehmigung zur Ausführung und Abrechnung kernspintomografischer Leis- tungen nach der Kernspintomografie-Vereinbarung (KernspinV) und der Qualitätssicherungsvereinbarung zur MR-Angiografie (MR-AngioV) haben. Die Tatsache, dass sich das LSG Berlin-Brandenburg mit dieser Rechtsfrage auseinandersetzen musste verwundert etwas, da bereits das Bundesverfassungsgerichts (BVerfG) in letzter Instanz in zwei Entscheidungen die Frage der Erbringung und Abrechnung von magnetresonanztomografischen Leistungen in der vertragsärztlichen Versorgung geklärt hatte.

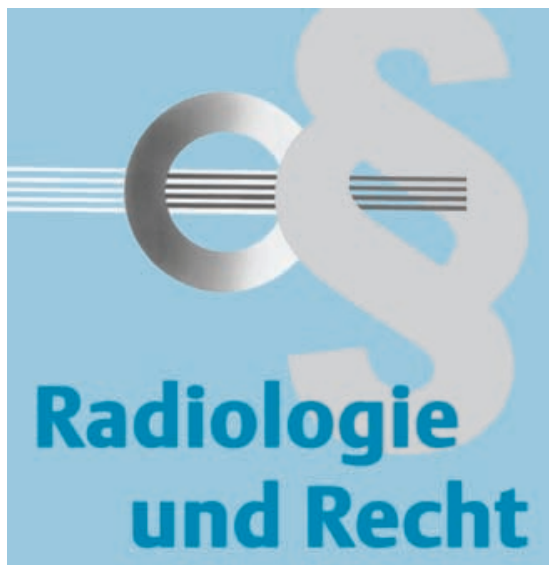

Bereits im Jahr 2004 hatte das BVerfG die Verfassungsbeschwerde eines Orthopäden gegen das Urteil des Bundessozialgerichts (BSG) zurückgewiesen, der gegen die Regelung in der KernspinV geklagt hatte, wonach die Erbringung von MRTLeistungen in der vertragsärztlichen Ver- 
sorgung ausschließlich Radiologen vorbehalten ist. Obwohl diese Frage damit sowohl in sozial-, als auch in verfassungsrechtlicher Hinsicht abschließend beurteilt worden war, musste sich das BSG im Jahre 2006 mit dem gleichen Anspruch eines Kardiologen auseinandersetzen, der sich als besonders qualifiziert zur Erbringung von MRT-Leistungen ansah. Trotz negativer Entscheidung des BSG, legte der Kardiologe eine Verfassungsbeschwerde beim BVerfG ein, die jedoch durch Gerichtsbeschluss vom 08.07.2010 nicht zur Entscheidung angenommen wurde, da ihr keine grundsätzliche Bedeutung zukam und sie darüber hinaus unbegründet war.

\section{Vorinstanz: \\ Urteil des SG Berlin 11.02.2004 $\nabla$}

Obwohl das BVerfG in der Entscheidung vom 08.07.2010 noch festgestellt hatte, „dass auch zur Durchführung der Kernspintomografie besonders qualifizierte Kardiologen, die die Voraussetzungen der fachlichen Befähigung gemäß § 4 Abs. 1 KernspinV nicht erfüllen, nicht ausnahmsweise zur Ausführung und Abrechnung kernspintomografischer Untersuchungen des Herzens im Rahmen der vertragsärztlichen Versorgung zugelassen werden müssen", hatte das Sozialgericht Berlin mit Urteil vom 06.04.2011 (Az.: S 71 KA 151/10) bei demselben Kardiologen überraschend die Auffassung vertreten, dass MRT-Untersuchungen des Herzens nicht nur von Radiologen, sondern auch von Kardiologen in der gesetzlichen Krankenversicherung durchgeführt und abgerechnet werden dürfen.

Die Entscheidung des SG Berlins war insbesondere deshalb auf Unverständnis gestoßen, weil die Urteilsgründe im krassen Widerspruch zu der Entscheidung des BVerfG stehen. Das SG Berlin hatte u.a. ausgeführt:

„Die Spezialisierung des medizinischen Fachwissens ist mittlerweile so weit fortgeschritten, dass die durch MRT-Untersuchungen gewonnenen Bilder, insbesondere Bewegungsbilder und Funktionsanalysen, fachgerecht nur noch durch den Facharzt mit dem entsprechenden Spezialwissen im Bezug auf das untersuchte Organ interpretiert werden können. Genau dieses Spezialwissen ist Gegenstand der Ausbildung zum Kardiologen, nicht aber Gegenstand der Ausbildung zum allgemei- nen Radiologen. Die Kammer schließt sich den Darstellungen des Klägers an, nach denen eine Interpretation der MRT-Befunde des Herzens tiefgehende Kenntnisse in der Pathoanatomie, der Pathophysiologie von Herz-Kreislauferkrankungen und der klinischen Kardiologie erfordern. Der Kläger hat auch weiter überzeugend dargelegt, dass das besondere Fachwissen der Kardiologen ein wesentlicher Grund dafür ist, dass Fort- und Weiterbildungsveranstaltungen für Kardio-MRT regelmäßig von Kardiologen für Radiologen abgehalten werden (...).“

Die Argumentation des SG Berlins war Gegenstand einer Stellungnahme der AG Herz- und Gefäßdiagnostik, die im Rahmen eines Sonderdrucks der RöFo „Zum aktuellen Standort der Radiologie in der Patientenversorgung“ im Juni 2011 zum 92. Deutschen Röntgenkongress erschienen war. Die AG Herz- und Gefäßdiagnostik hatte sowohl der Urteilsbegründung, als auch der Interpretation des klagenden Kardiologen inhaltlich und fachlich mit zutreffenden Argumenten widersprochen:

1. Die Ausbildung in kardialer Bildgebung (CT, MRT) ist integraler Bestandteil der radiologischen Weiterbildung. In der Ausbildung zum Kardiologen dagegen ist die Vermittlung von Spezialwissen auf dem Gebiet der MRT und CT des Herzens nicht enthalten.

2. Die DRG verfügt schon seit mehr als 10 Jahren über eine strukturiertes Fortbildungsprogramm und ein internes Qualifizierungs- und Qualitätssicherungssystem. Radiologen suchen in der Herzbildgebung zweifelsfrei die Interaktion mit Kardiologen; daraus darf aber nicht abgeleitet werden, dass ein Kardiologe für die Fortbildung von Radiologen besonders geeignet oder gar erforderlich ist. Genau das Gegenteil ist der Fall: ein Kardiologe, der die Zusatzqualifikation „MRT des Herzens“ erwerben möchte, muss nach der gültigen Rechtslage die Weiterbildung bei einem Radiologen nachweisen.

3. Der klinische Erfolg der Methode beruht in erster Linie auf technischen Weiterentwicklungen. Gerade kardiale MRT-Untersuchungen stellen bereits bei der Durchführung eine besondere Herausforderung dar, weil das Protokoll und Messparameter für viele Patienten individuell angepasst werden müssen. Diese hohe technische Kompetenz in der MRT ist we- sentlicher Bestandteil der radiologischen Weiterbildung und ein Alleinstellungsmerkmal radiologischer Fachärzte.

4. Die generelle Einschätzung, dass klinisches Spezialwissen in Bezug auf das untersuchte Organ, dass nur in den entsprechenden klinischen Weiterbildungen vermittelt wird, für die Interpretation radiologischer Bilder zwingend erforderlich ist, ist falsch. Vielmehr zeichnet sich die Kompetenz des Radiologen heute dadurch aus, dass sowohl klinisches Fachwissen, als auch methodische Expertise für bildgebende diagnostische Verfahren in den diagnostischen Prozess einfließen und auf vielen Gebieten der Medizin in hohem Maße zur schnellen und zielgenauen Diagnostik und Therapie der Patienten beitragen.

5. Bei jeder kardialen MRT werden andere Organe (Lunge, Mediastinum, Mamma, große Gefäße, Skelett/Wirbelsäule, Oberbauch) miterfasst, die dann auch mit beurteilt werden müssen. Die umfassende Ausbildung zur Beurteilung aller Strukturen ist integraler Bestandteil der radiologischen Weiterbildung und ein weiteres Alleinstellungsmerkmal radiologischer Fachärzte.

6. Die Trennung zwischen bildgebender Diagnostik und klinischer Patientenversorgung macht auch wirtschaftlich Sinn, da sie kostentreibende Selbstzuweisungen verhindert. In der jüngst verfügbaren Übersicht des Europäischen Kardio-CT-Registers der ESC erreicht die Selbstzuweisungsrate der Kardiologen $60 \%$ und mehr.

\section{Urteil des Landessozialgerichts Berlin-Brandenburg \\ $\nabla$}

Das LSG Berlin-Brandenburg hat mit seinem Urteil vom 20.02.2013 das Urteil des SG Berlin aufgehoben und die Klage des Kardiologen abgewiesen. In seiner Urteilsbegründung ist das LSG den Argumenten der bisherigen Rechtsprechung des BSG und des BVerfG in vollem Umfang gefolgt.

Im Einzelnen stellte das LSG folgendes fest:

- Bedeutung der Zusatzbezeichnung "fachgebundene MRT“

Der Erteilung einer Abrechnungsgenehmigung für Kardiologen steht zunächst entgegen, dass nach $§ 4$ Abs. 1 
Nr. 2 KernspinV und $\S 3$ Abs. 1 Nr. 2 MR-AngioV ausschließlich Ärzte, die die Facharztbezeichnung „Radiologie“ führen, berechtigt sind, eine Genehmigung unter den dort genannten Voraussetzungen zu erhalten. Die Vorschriften der KernspinV und der MRAngioV können auch nicht dahin ausgelegt werden, dass anstelle der genannten Facharztqualifikationen die erworbene Zusatzbezeichnung „fachgebundene MRT“ genügt. Damit hat das LSG deutlich gemacht, dass die Zusatzbezeichnung „fachgebundene MRT" keine ausreichende fachliche Qualifikation darstellt, um hierüber anderen Fachgebieten als Radiologen eine Abrechnungsgenehmigung für MRT-Leistungen in der GKV nach $\S$ 135 Abs. 2 SGB V einzuräumen. Begründet hat das LSG seine Auffassung einerseits damit, dass landesrechtliche Regelungen die zum Erwerb der Zusatzbezeichnung verlangt werden, nicht bundesweit, sondern nur in 15 von 16 Landesärztekammern existieren. Auch hätten die Partner der Bundesmantelverträge bewusst davon abgesehen, anstelle der in $\S 4$ Abs. $1 \mathrm{Nr} .2$ KernspinV und $\S 3$ Abs. 1 Nr. 2 MR-AngioV aufgeführten Facharztabschlüsse auch die Fachärzten anderer Gebiete erteilte Zusatzbezeichnung für fachgebundene MRT ausreichen zu lassen.

- Kein verfassungsrechtlicher Anspruch von anderen Ärzten auf Gleichbehandlung nach Art. 3 Abs. 1 GG Nach Ansicht des LSG sind Fachärzte, die über keine der in $\S 4$ Abs. 1 Nr. 2 KernspinV genannten Abschlüsse, wohl aber über eine Zusatzqualifikation für fachgebundene MRT verfügen, auch nicht aus verfassungsrechtlichen Gründen gleichzustellen, da es sachliche Gründe im Sinne des Gleichbehandlungsgrundsatzes nach Art. 3 Abs. 1 Grundgesetz (GG) gebe, die eine Differenzierung rechtfertigen würden. Der Gesetzgeber habe mit der Vorschrift des $\S 135$ Abs. 2 Satz 4 SGB V den Vertragspartnern die Möglichkeit gegeben, die Durchführung von technischen Leistungen auf die Fachärzte zu konzentrieren, für die diese Leistungen nicht nur zum Rand, sondern zum Kern ihres Fachgebiets gehören, d. h. für ihr Gebiet wesentlich und prägend sind, um die Qualität und Wirtschaftlichkeit der Leistungen zu verbessern. Insbesondere sollte den Vertragspartnern eine Leistungssteuerung ermöglicht werden, die eine Trennung zwischen der Diagnosestellung und Befundbewertung durch den therapeutisch tätigen Arzt einerseits und der Durchführung der diagnostischen Maßnahmen (medizinisch-technischen Leistungen) durch den lediglich diagnostisch tätigen Facharzt andererseits bewirke. Die Konzentration der Leistungserbringung im Bereich der Kernspintomografie auf das Fachgebiet der Radiologie gewährleiste, dass die für die spezifische medizinische Fragestellung am besten geeignete diagnostische Methode ausgewählt werde und die Ergebnisse sachgerecht interpretiert würden, z. B. sog. Zufallsbefunde erkannt würden. Außerdem bewirke eine derartige Arbeitsteilung im Sinne des sogenannten Mehraugenprinzips, dass die Diagnostik unabhängig von einem eventuellen Interesse an der Therapie erfolge, damit der optimalen $\mathrm{Pa}-$ tientenversorgung diene und außerdem dem sparsamen Einsatz der Leistungsressourcen.

Die Regelung führe zu einer wirtschaftlicheren Leistungserbringung, da die Gefahr einer überproportionalen Leistungsausweitung darin bestehe, dass therapeutisch tätige Fachärzte der sog. Organfächer aufwändige diagnostische Maßnahmen, wie z.B. ein MRT, selbst durchführen, anstatt sie hierfür schon durch ihre Facharztqualifikation befähigten Vertretern der sog. Methodenfächer zu übertragen. Demgegenüber würden Arztgruppen der sog. Methodenfächer dem Überweisungsvorbehalt und den Beschränkungen des Zielauftrags unterliegen. Beides verhindere weitgehend, dass Mitglieder dieser Arztgruppen allein durch eigenes Zutun ihre Leistungsmenge ausweiten könnten.

- Gefahr einer überproportionalen Leistungsausweitung

Das LSG hat damit die Argumentation, die bisher in mehreren Urteilen seitens des BSG und des BVerfG vertreten worden ist, aufgenommen und bestätigt. Es ist der Auffassung der Krankenkassen gefolgt, die in dem Verfahren vorgebracht hatten, dass „,bei einer Öffnung der MRT-Diagnostik für andere klinische Disziplinen mit einer Beeinflussung der Wirtschaftlichkeit im Sinne einer überproportionalen Leistungsausweitung durch vermehrte Selbstüberweisung zu rechnen“ sei. An Letzteres haben nach Ansicht des LSG die Partner der Bundesmantelverträge angeknüpft, indem sie in nach- vollziehbarer Weise die Gefahr einer überproportionalen Leistungsausweitung darin erkannten, dass therapeutisch tätige Fachärzte der sog. Organfächer aufwändige diagnostische Maßnahmen, wie z.B. ein MRT, selbst durchführen, anstatt sie hierfür schon durch ihre Facharztqualifikation befähigten Vertretern der sog. Methodenfächer zu übertragen. Demgegenüber unterliegen Arztgruppen der sog. Methodenfächer, wie z. B. Laborärzte, Nuklearmediziner, Pathologen und Radiologen, dem Überweisungsvorbehalt $(\S$ 13 Abs.4 Bundesmantelvertrag-Ärzte BMV-Ä bzw. § 7 Abs.4 Arzt-Ersatzkassenvertrag - EKV) und den Beschränkungen des Zielauftrags ( $§ 24$ Abs. 72 Nr. 2 BMV-Ä und $\S 27$ Abs. 71 Nr. 2 EKV). Beides verhindere weitgehend, dass Mitglieder dieser Arztgruppen allein durch eigenes Zutun ihre Leistungsmenge ausweiten könnten (Wigge NZS 2005, 176).

Diesen Gesichtspunkten komme bei medizinisch-technischen Leistungen, die typischerweise sowohl kostspielig seien als auch für den Patienten belastend sein können, wie z.B. Computeroder Magnetresonanztomografie, besonders große Bedeutung zu. Die Regelung in $\S 135$ Abs. 2 Satz 4 SGB V diene deshalb sowohl der Gesundheit der Versicherten als auch der finanziellen Stabilität und Funktionsfähigkeit der gesetzlichen Krankenversicherung (Begründung zum Entwurf des GMG, BT-Drs. 15/1525, S. 124).

- Keine Beschränkung der Genehmigung auf einzelne Organe

Die KernspinV sehe darüber hinaus nur eine Genehmigung zur Ausführung und Abrechnung aller kernspintomografischen Untersuchungen und eben keine Beschränkung auf MRTLeistungen im Bereich eines einzelnen Organs vor. Durch $\S 4$ Abs. 1 Nr. 2 KernspinV haben die Partner der Bundesmantelverträge nach Auffassung des LSG im Einklang mit der Ermächtigungsnorm des $\S 135$ Abs. 2 Satz 4 SGB V zum Ausdruck gebracht, dass die kernspintomografische Diagnostik grundsätzlich bei den hierfür qualifizierten Ärzten für Radiologie konzentriert werden und sie nicht den Ärzten offen stehen soll, die - wie der Kläger - zu diesem diagnostischen Verfahren nur in einem Teilbereich, z. B. beschränkt auf ein einzelnes Organ, befähigt sind. 
- Kein Eingriff in die Berufsfreiheit aus Art. 12 Abs. 1 GG

Der Abrechnungsausschluss verstoße auch nicht gegen das Grundrecht der Berufsfreiheit nach Art. 12 Abs. 1 GG, da Kardiologen durch die Regelung nicht in ihrem Status, sondern allenfalls in einem Teilausschnitt ihrer ärztlichen Tätigkeit betroffen seien und die Regelung dazu diene, die Qualität der Versorgung sowie der Wirtschaftlichkeit im Interesse der Funktionsfähigkeit der gesetzlichen Krankenversicherung zu erhalten. Mit dieser Argumentation greift das LSG vollständig auf die Ausführungen des BVerfG zurück, welches in seinem Beschluss vom 08.07.2010 bereits die Auffassung vertreten hatte, dass es in dem vorliegenden Verfahren weder um den Zugang zu einer bestimmten Arztgruppe noch zu einem Planungsbereich, sondern lediglich um die Abrechenbarkeit bestimmter Leistungen zulasten der gesetzlichen Krankenversicherung gehe. Ein Arzt werde jedenfalls so lange nicht in seinem Status betroffen, wie er nicht im Kernbereich seines Fachgebiets eingeschränkt werde.

Der Kläger werde allenfalls in einem Teilausschnitt seiner ärztlichen Tätigkeit betroffen. Denn derzeit zähle die Durchführung von MRT-Untersuchungen weder nach dem Recht der Ärzte- kammer Berlin noch nach der von der Bundesärztekammer verabschiedeten Musterweiterbildungsordnung bzw. den -richtlinien zu den Inhalten einer Tätigkeit als Facharzt für Innere Medizin mit dem Schwerpunkt Kardiologie. Der Kläger habe auch nicht dargelegt, dass es ihm wirtschaftlich oder in sachlicher Hinsicht unzumutbar wäre, die kernspintomografische Diagnostik bei gesetzlich Versicherten durch einen Radiologen vornehmen zu lassen. Schließlich seien die Regelungen in der KerspinV und der MR-AngioV nicht deshalb ungeeignet, weil Radiologen für die Durchführung kernspintomografischer Untersuchungen des Herzens einer speziellen Fortbildung bedürften. Auch Kardiologen müssten sich im Regelfall einer solchen Fortbildung unterziehen, zumindest insofern, als ihnen umgekehrt Kenntnisse der Kernspintomografie typischerweise fehlen werden. Da die Konzentration aller kernspintomografischen Leistungen bei den Radiologen ferner dazu beitragen soll, die diagnostisch tätigen Ärzte als Berufsgruppe zu erhalten, war vorliegend hinsichtlich des zur Herzdiagnostik mittels Kernspintomografie u.U. besonders qualifizierten Klägers keine andere Betrachtung geboten.
- Ergebnis

Das LSG Berlin-Brandenburg widerspricht damit deutlich der Auffassung des SG Berlin, welches in der Vorinstanz mit Urteil vom 06.04.2011 die Auffassung vertreten hatte, dass Kardiologen einen Anspruch auf Erteilung einer Abrechnungsgenehmigung hätten, soweit sie die Anforderungen der KernspinV und der MR-AngioV erfüllen würden. Mit dem Urteil wird in zutreffender Weise die Rechtsprechung des BSG vom 11.10.2006 (B 6 KA 1/05 R) und des BVerfG vom 08.07.2010 (Az.: 2 BvR 520/07) bestätigt. Beide Gerichte hatten über denselben Sachverhalt bereits mit dem gleichen Ergebnis wie jetzt das LSG, entschieden.

RA Dr. Peter Wigge

Fachanwalt für Medizinrecht

Rechtsanwälte Wigge

Scharnhorststr. 40

48151 Münster

Tel.: (0251) 53595-0

Fax: (0251) 53595-99

E-Mail: kanzlei@ra-wigge.de

Internet: www.ra-wigge.de 